\title{
Implementing the Component-based Software Engineering in Embedded Systems
}

\author{
Mohammed A. Abdallah \\ Department of Electrical and Computer Engineering \\ Tennessee Technological University, Cookeville, TN 38501, USA \\ masadik21@tntech.edu
}

\begin{abstract}
It is thought that the component-based software engineering can not be used in the embedded systems. This thought is the result of the features of embedded systems. Embedded systems have some unique concerns such as resource constraints, real-time or dependability requirements. These issues make using the component-based software engineering into the embedded systems field more difficult. The aim of this work is to show that it possible to make use of component-based concept in the embedded systems. This paper considers the basic overview of component-based model and general issues about embedded systems. The core issue is providing an example showing that how useful to implement an embedded system using the component-based software engineering.
\end{abstract}

\section{INTRODUCTION}

Component-based software engineering becomes a new approach in software development. The idea behind the component-based approach is designing the desired system in terms of components. The advantage of the component-based approach is providing the reusability of these components. Moreover, if the component is the logical unit of work, maintenance, testing and updating systems using these components will be easy and fast. Component-based approach has been largely used for several years in desktop applications, office applications, e-business and web-based distributed applications. But it is noticed that in other environments such as embedded and real-time systems, component-based approach has less utilization. It has been shown that using the component technology in some environments is difficult because of system requirements and constraints [1].

Traditional desktop applications are limited in many applications. Desktop devices are large and need a particular care to be maintained well and to be integrated with the environment that needs the service. There are other devices such as embedded devices that are small and do the same function as the personal computer (PC) but they can be embedded inside the environment easily. An embedded system is a special-purpose computer system, which is completely encapsulated by the device it controls. An embedded system has specific requirements and performs predefined tasks, unlike a general-purpose personal computer [1]. These embedded systems can also be considered as real-time systems. They must meet stringent specifications for safety, reliability, limited hardware capacity and low power. This may lead to increase the complexity of these systems. This increased complexity leads to necessary achieve some issues such as: requirements engineering, high-level design, early error detection, productivity, integration, verification and maintenance $[2,3]$.

It can be concluded from the previous paragraphs that component-based approach is an efficient way to build complex systems and the embedded systems are used in many files and machines nowadays which is run-time environments. So, if the component-based approach is used in building and designing these embedded systems, many advantages will be achieved. Time to build an embedded system will be reduced, easy testing will be provided and easy and fast system maintenance. Unfortunately, the speed of developing embedded system using the component-based approach is very slow due to the restrictions of these real-time systems. These restrictions are the memory size of the embedded systems, the low power and the small size [2].

In this paper, an overview of the component-based software engineering will be introduced and a case study of a research embedded system that makes use of the component-based approach. This paper also shows the benefits that are achieved due to employment the components into the embedded system. This paper is organized as follows. Section 2 gives an overview of the component-based software engineering, section 3 considers the case study about a research embedded system project and section 4 illustrates the implementation of the embedded system. Section 5 shows some of the experimental results. Finally, the conclusion of this paper and the future work are introduced.

\section{COMPONENT-BASED SOFTWARE ENGINEERING}

This approach mainly depends on building systems from existing components, providing support for the development of systems as assemblies of components. It is also supporting the development of components as reusable units and it is facilitating the maintenance and evolution of systems by customizing and replacing their components [4]. The component-based approach has some advantages over the traditional programming. Firstly, 
advantages from the business point of view in terms of shorter time-to-market, lower development and maintenance costs. Secondly, advantages from technical and engineering point of view which can be increased understability of complex systems and increased the reusability, interoperability, flexibility, adaptability, dependability. Thirdly, they have advantages from strategic point of view of a society such as increasing software market, generation of new companies. Finally, component-based approach has been successful in many application environments [4].

\section{A. Basic Definitions in Component-based Approach}

The basis logical unit of work is the component. Components can be assembled according to the rules specified by the component model. Components are assembled through their interfaces. A Component Composition is the process of assembling components to form an assembly, a larger component or an application. Components are performing in the context of a component framework. A component technology is a concrete implementation of a component model.

A software component is a software element that confirms a component model, can be independently deployed and composed without modification according to a composition standard. A component model defines specific interaction and composition standards [5].

Components can be described in different ways like white, grey or black box. Precondition is an assertion that the component assumes to be fulfilled before an operation is invoked. Postcondition is an assertion that the component guarantees will hold just after an operation has been invoked, provided the operation's pre-conditions were true when it was invoked. Invariant is a predicate over the interface's state model that will always hold [6].

\section{B. Basic Principles of the Component-based Software Engineering}

Reusability. It means that the same component can be used in many systems. The desire to reuse a component leads to some technical constraints such as: good documentation should be available to be able to reuse a component as well as a well-organized reuse process and the similar architecture of the components should be provided to ensure the consistency and the efficiency of the system [7].

Substitutability. The overall system should work in spite of which component is used. There are some limitations in this area such as: the runtime replacement of the components [7].

Extensibility. Extensibility can take one of two shapes either extending components that are part of a system or increase the functionality of individual components. But there are technical challenges such as: design-time [7].

\section{CASE STUDY}

In this section, a research system is introduced without implementing it via the component-based approach. A general overview on this system is introduced to know the purpose of this system and where it can be useful. This system depends on the system - on-a-chip design technology. Systems-on-a-chip design technology provide the computational power to eliminate the need of a computer and interfaces by providing integrated design of processors, on chip memory, and peripheral controllers. State of the art advances in integrated circuit technology, especially in the programmable logic devices, initiated the era of Systems-on-Programmable-Chips. Field Programmable Gate Arrays (FPGA) are modern, complex programmable logic devices (chips) that are manufactured with a high density of configurable blocks [8].

\section{A. The Purpose of the System}

This system is considered as a data acquisition system. It can capture analog signals from an environment such as industrial or medical environments without using a PC. Then these analog signals are converted into digital form and then go to some processing such as filtering or amplification. In addition to the capability of plotting these original signals in a Liquid Crystal Module (LCM) for monitoring or testing. This system is designed using the latest advances in FPGA chip design. It eliminates the need of a personal computer and the cumbersome and expensive data acquisition systems currently in use in the field [9]. The overall system is shown in figure 1. As shown in this figure, the FPGA board is able to capture the signals and then all signal analysis and processing are take place inside the FPGA. Finally, the original signals can be plotted into this LCM in the right hand side of Fig. 1.

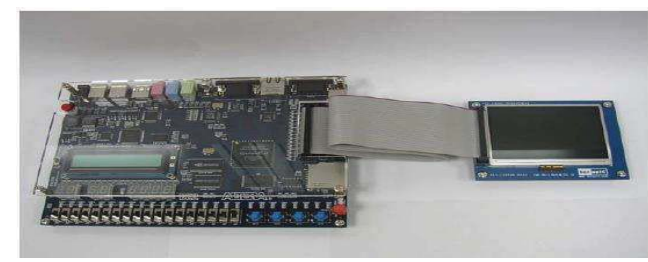

Figure 1. The overall system

\section{B. Implementation of the System using Traditional Programming}

Figure 2 shows how the desired system is implemented via the traditional programming. The language that is used to implement this system is the Verilog. The IDE that used is the Altera Quartus II. The whole code is written in the single module. 


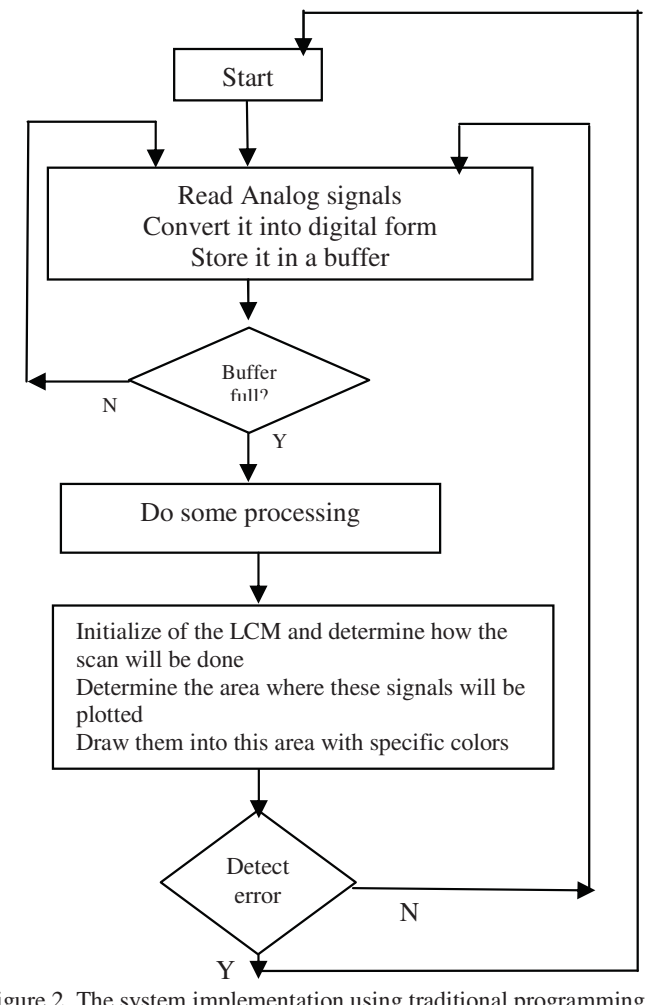

Figure 2. The system implementation using traditional programming

\section{COMPONENT-BASED IMPLEMENTATION}

As we can see in figure 2 above, there are many functions listed in the same block and all these blocks were written in the same module. So, this single module is the only responsible of the total task of the system. If any error is occurs, the designer can not know easily where the location of the error because the whole module is not working although there is one error in a specific part in this module which is faulty. So, if we can collect the functions that do a single kind task in single module, it will be easier to detect the location of the error knowing the nature of it. These collected functions can be done in a component. So, a single component is responsible for a single task and then it is necessary to integrate these components together via interfaces to build the total system. This idea can be supported in Altera Quartus II using Verilog language. This language deals with modules so, each component is considered as module do a specified task. It is needed to design a top level module that will integrate or woven these components together to achieve the total system requirements in efficient way. Figure 3 illustrates how the traditional programming can be handled in more efficient way if it deals with components. Each block now is corresponding to a single component which in turn is mapped to a single module.

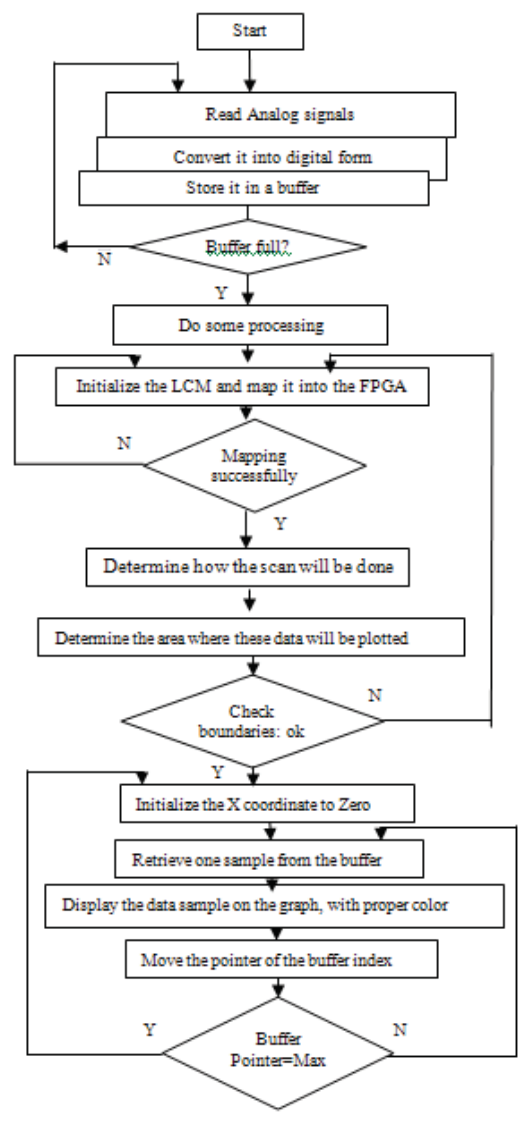

Figure 3. The system implementation using component-based approach

A. Implementation of the System using SOPC and NIOS II Processor

Another method to implement the same system is using the System on Programmable Chip (SOPC). SOPC is a part of Altera Quartus II. Its function is taking a module that is written in a Verilog language and result a component which has an input and an output and all its internal contents is hiding. Then SOPC will automatically generate a system which contains many components and incorporate between them. This generated system is passed to another IDE called NIOSII. The NIOS-II processor core is connected to its components through the Avalon switch interface. The Avalon interface contains logic and the arbitration to manage this connection. The system components are specified in a graphical user interface (GUI) and once the system is generated all the necessary logic is automatically created in the form of HDL files [10]. The hardware language description from the user side is limited to building the top-level module where the NIOS-II processor is instantiated. The NIOS-II processor is a 32-bit RISC processor. It has a 4-kB instruction cache 


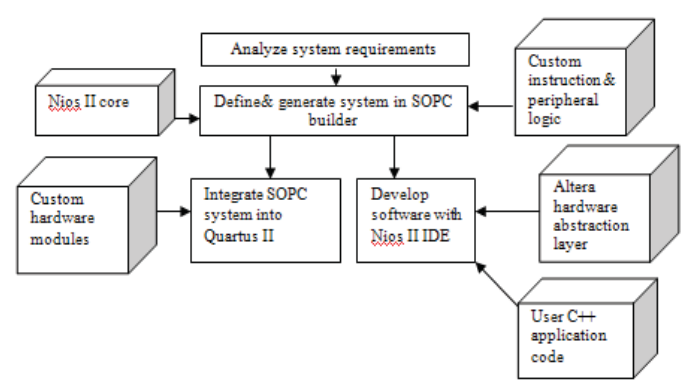

Figure 4. The system implementation using component-based approach via SOPC and NIOS II processor

and a $2-\mathrm{kB}$ data cache. The processor runs on a clock of 50 $\mathrm{MHz}$. Since the NIOS-II processor follows Harvard architecture the data and the program are stored separately. Figure 4 shows how to implement the desired system using the SOPC and the NIOS II Processor.

\section{EXPERIMENTAL RESULTS}

Figure 5 shows the curve that is displayed on the LCM using the FPGA without using a computer. It is shown in this figure that there are two axes that are drawn in red and the curve in red that are corresponding to the data values.

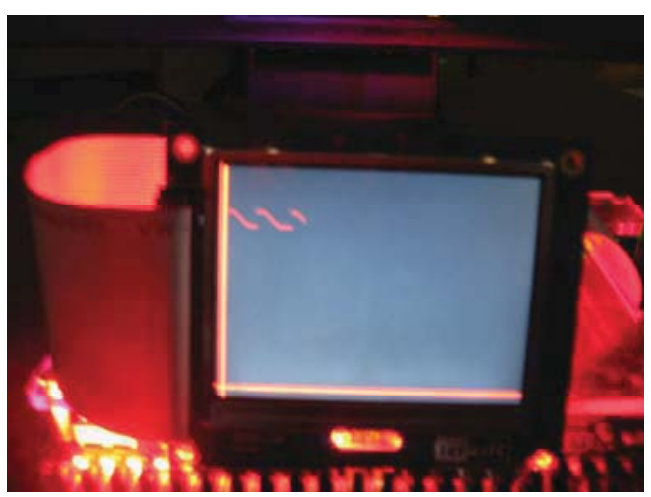

Figure 5. Experimental data plots using the FPGA module

\section{CONCLUSION}

Component-based software engineering becomes a new approach in software development. Embedded systems are used in many fields in our life. So, if the component-based approach is used in building and designing these embedded systems, many advantages will be achieved. Time to build an embedded system will be reduced, easy testing and fast system maintenance and updating. In this paper, an example of how to use the component-based software engineering in designing the embedded system is introduced. It can be concluded that hardware language (e.g. Verilog) designers are not professional in writing a code in efficient way. So, if there are standards that help designers of embedded system to deal with components, the generated systems will be more efficient and powerful. The development of using the component-based approach in embedded systems is still slow. This area of research needs a lot of work to develop standards to work with in designing process and dealing with components in many programming fields.

\section{FUTURE WORK}

This work which considered the component-based software engineering can be extended to consider the aspect approach. The main idea in the aspect approach is to determine the core concerns of the system and implement the cross-cut concerns separately from the core concerns.

\section{ACKNOWLEDGMENT}

I would like to acknowledge Dr. Elkeelany and Dr. Siraj for their support to this work. Also, I would like to acknowledge the center of Manufacturing Research (CMR) at Tennessee Tech University, for its support of this research.

\section{REFERENCES}

[1] Ivica Crnkovic, "Component-based Software Engineering for Embedded Systems," $27^{\text {th }}$ International Conference on Software Engineering, ICSE'05, ACM, St. Luis, USA, May, 2005

[2] Ivica Crnkovic, Jakob Axelsson, Susanne Graf, Magnus Larsson , Rob van Ommering, Kurt Wallnau, "COTS Component-Based Embedded Systems - A Dream or Reality?," 4th International Conference, ICCBSS 2005, Springer, Bilbao, Spain, February, 2005

[3] Pimentel,B.S; de Aliva Valgas Filho, J.H;Campos, R.L;Fernandez,A.O, Nunes Coelho, C.J ,“A FPGA implementation of a DCT-based digital electrocardiographic signal compression device," IEEE,14th Symposium on Integrated Circuits and System Design,2001.

[4] Ivica Crnkovic,Heinz Schmidt, Judith Stafford, Kurt Wallnau, "Rule-driven component composition for embedded systems," proceedings $4^{\text {th }}$ ICSE workshop on component-based software engineering, Canada, May,2001

[5] G.Heineman,W.Councel, “ Component-based Software engineering ,Putting the pieces together," Addoson Wesley,2001

[6] Uwe Asmann, Holger Bar, Thomas Gensler, "Meta-Programming Grey-Box Connectors," IEEE Computer Society ,p. 300, 2000

[7] Ivica Crnkovic, Magnus Larsson, "Building reliable componentbased software systems," Artech House publisher, 2002

[8] Emil J., Gelabert P., Adhami R., Wheelock B. and Adams R., "Real Time Holter Monitoring of Biomedical Signals", DSP Technology and Education Conference DSPS'99, Houston, Texas, 1999

[9] O. Elkeelany, M. Abdallah, A. Alouani, "An Efficient Embedded System Design for Capturing and Storing Analog Data," Journal of Engineering and Applied Sciences, Medwell Journals, vol. 2(8), pp. 1290-1296, 2007

[10] Quartus II Version 6.0 Handbook, Volume 4, SOPC Builder, Altera, November 2006 